\title{
Families influence your health!
}

\author{
Ursula Dietrich
}

Published online: 8 August 2014

(C) Springer-Verlag Berlin Heidelberg 2014

The world population is facing severe challenges for its health care systems and the distribution of medical care in general. The articles in this issue cover health across the entire lifespan and focuses on the influence of economic factors on health. The articles address the influence of parental health behaviour on their children, as well as behaviour-based risk factors towards chronic disease among adolescents. Another question that is raised is concerned with the care given to elderly patients with dementia.

The care of elderly people in need of assistance is affected by demographic changes and therefore will increasingly gain importance. In addition to home care provided by members of a family, the institutionalized care and medical assistance for people suffering from dementia is an important factor. Kuske et al. carried out a systematic review trying to identify potential aspects that need improvement in the context of security relevant connections and the exchange of relevant information between different health care settings. Again, further research is required as there currently is a lack of sound evidence especially concerning the support of people with dementia. The work strains which can affect the nursing staff (e.g. in terms of somatic complaints, mental impairment) in German inpatient geriatric care were analyzed by Dietrich et al. They identified some influential factors and starting points for measures to reduce the level of subjectively experienced burdens and to support the work ability and motivation of the care staff. The results of both studies contribute towards the improvement of care for people with the dementia.

Erkelenz et al. found out that the health-related behaviour of parents and their motivation towards physical activity,

U. Dietrich $(\square)$

Research Association Public Health Saxony, Faculty of Medicine Carl Gustav Carus, Technische Universität Dresden, Fiedlerstrasse 33, D-01307 Dresden, Germany

e-mail: Ursula.Dietrich@tu-dresden.de especially that of mothers, influences the physical fitness of their children. This study, which was conducted in Germany, could show that there is a relation between socioeconomic variables of the family and the physical fitness of children of said families. A second study that focused on the influence of parents on the health of their children was presented by Labree et al. who researched to find a connection between the BMI, socioeconomic factors and the migrational background of parents and the BMI as well as the condition of overweight as pertains to their children who attend school in the Netherlands. They concluded that the BMI of parents is a significant predictor for the BMI of their children. Skranes et al. looked into the question of how Norwegian mothers use the Internet to find out about health-related questions they have concerning their young children in general and in times of sickness. Several factors were analysed. However, the actual influence of such informational sources and their influence on behavioural change, self-management capabilities and the decision-making process of parents requires further research. The impact of these factors on the health of children also needs additional studies. Smpokos et al. studied the influence of five clustered behavioural risk factors for chronic disease on several biological factors and the cardiorespiratory fitness among Greek adolescents. Based on the results of this study, recommendations for health promotion programs for adolescents were created and the results strongly support the aspect of including parents in the development of health-promotion programs for children.

Another aspect of this issue puts the focus on studies researching the socioeconomic background. Diederich et al. showed that some behaviors which are regarded as risky lead to the inclusion of additional payments for medical treatment within Germany.

The prevalence of zinc deficiency among a semi-urban community, its relation to the socioeconomic status and 
several indices of the metabolic syndrome were the subject of investigation of Awobusuyi et al. Mostafavi et al. evaluated relevant health behaviours of homeless people in Teheran. Gender specific differences were found and the outcome of this study underlines the importance of socioeconomic factors towards health and the acceptance of offers which aim to maintain or improve ones health.

In line with this topic is the article written by Baiden et al. who researched the correlation between the feeling of belonging to a group and unmet health care needs. This study shows the importance of a supportive environment by enhancing community resources and appropriate access to community-based health prevention programs.

The result of this issue underlines the importance of positive familiar influences as well as health care policies and health promotion programs that need to be tailored to the specific needs of different target groups (gender specific, socioeconomic and cultural) and improved accessibility to these programs. 